\title{
The Validation of Monte Carlo Methods for Scanning Electron Microscopy and Electron Microprobe Analysis
}

\author{
E. Lifshin, Bradley Thiel and Raynald Gauvin*
}

College of Nanoscale Science and Engineering, University at Albany, 251 Fuller Road, Albany NY 12203

*Department of Mining, Metals and Meterials Engineering McGill University, Montreal, Canada, H3A 2B2

Monte Carlo methods are well recognized as an effective way to simulate the interaction of an electron beam with a solid and thereby predict both the signals generated from samples for analysis and the volumes from which those signals are generated $[1,2]$. In the case of electrons, information calculated includes the number of backscattered electrons, the energy and spatial distribution of those electrons, and the depth distribution from which those electrons originate. Similar information can be obtained for secondary electron emission and transmitted electron signals. For x-rays, complete spectra can be predicted including the continuum and characteristic lines as well as the corresponding depth distribution functions for $\mathrm{x}$-ray generation, $\phi(\rho z)$.

In looking back on the history of electron beam microanalysis one of the first approaches to quantifying the signals generated was to apply the basic physics of electron scattering and energy loss, and the concepts of cross section and absorption to generate closed form analytical solutions that could be applied to signal characterization and quantitative analysis. Thus, we saw the emergence of the Philibert absorption correction, the Reed fluorescence correction and the Poole and Thomas atomic number corrections following Castaing's ground breaking work to describe the signals generated in the electron microprobe $[3,4]$. All of these theory based equations, which formed the basis of the ZAF (atomic number, absorption, fluorescence) correction procedure, however required some degree of tuning to provide the best match for a broad range of experimental data. The tuning took the form of adjustable parameters, the values of which were established by matching the model to a set of experimental data. The need for this approach is not surprising since often the models required assumptions that were not broadly valid or the equations used were simplified to obtain compact closed form solutions. Available computers did not have the horsepower to churn through the analysis of multicomponent systems in a reasonable amount of time. The simplification of the approach went even further by depending more on basic correlation of data with experiment. For example, the Philibert absorption equation was replaced by the Heinrich equation which describes the amount of absorption in materials with a combination of beam energy, mass absorption coefficients, excitation potential and the take-off angle of the emitted $\mathrm{x}$-rays [5]. Although the underlying physics is fairly hidden, this method works very well and is incorporated in a very large number of computer programs in use today. An even higher level of abstraction came in the replacement of ZAF procedures with $\phi(\rho z)$. While this method goes back to Castaing and the experimental roots of microanalysis, it depends heavily on curve fitting equations with parameters tuned to match some set of experimental data and the hope that those equations can be more broadly applied to any sample. In the case of quantitative electron microprobe analysis this has also worked reasonably well and checked with a set of data that exists for measured $\mathrm{K}$ ratios (intensity measured on a sample/intensity measured on a standard) for a large number of systems [6]. This data base has often been used to check the accuracy of quantitative analysis models. 
Although quantitative analysis of thick polished samples has been quite successful using existing analytical models, Monte Carlo methods for a long time have offered the promise of providing useful information in situations where conventional closed form solution models can not be easily applied. Examples include small particles either embedded or on a surface, rough surfaces, inclined specimens and thin films, although there are some $\phi(\rho z)$ methods that address the latter. The advantage of the Monte Carlo method is that not only does it allow for a return to the underlying physics of electron solid interactions, but it can handle geometrical situations that would be difficult or impossible by other means. The disadvantages include the large numbers calculations, particularly since quantitative microanalysis requires iteration, and the unfortunate fact that even with the most complete physical equations satisfactory matches with experimental data cannot always be achieved unless some adjustable parameters are present. While the first disadvantage may be to some extent overcome with faster computers, the second requires both a better understanding of the factors that influence electron solid interactions and better data to test the models. Thus, for example, a model that assumes samples are amorphous may lead to errors, as can assuming that the density of a thin film is the same as a bulk sample. Adjustable parameters are not strangers to Monte Carlo models and needs to be revisited in a logical manner. In the early work of Curgenven and Duncumb the impact parameter was selected to give the best fit with experimental backscatter data [7]. More recently, ionization cross sections have had to be adjusted to give a decent match with absolute $\mathrm{x}$-ray intensities even when $\mathrm{K}$ ratio predictions are accurate with such adjustments. The need therefore clearly exist to establish a set of accurate data in addition to electron backscatter coefficient variation with atomic number that will make it possible to improve Monte Carlo and other models. The components of this data base are yet to be established, but they certainly could include absolute $\mathrm{x}$-ray yields, relative line intensities from pure standards, measured $\mathrm{K}$ ratios for alloys and compounds, measured $\phi(\rho z), \mathrm{K}$ ratios as a function of film thickness for pure elements and multicomponent systems, curves of backscatter fraction as a function of atomic number and tilt along with energy distribution information, secondary electron yields as a function of material, and all of the data collected at a range of beam energies. While some of this data exists, a lot more needs to be collected and used for model refinement. In the case of Monte Carlo methods the goal is

reasonably clear, but the journey is only partially complete. In the meantime they will play more of a role of helping to assess analytical problems like estimating excitation volumes and detection limits than as a versatile method for accurate quantitative x-ray analysis for a broad range of samples and sample geometries as well as play a major role in understanding SEM resolution and contrast.

\section{References}

[1] Joy, D. C., Monte Carlo Modeling for Electron Microscopy and Microanalysis, (1995), Oxford University Press

[2] Heinrich, et. al., Use of Monte Carlo Calculations in Electron Microanalysis and Scanning Electron Microscopy, (1976), NBS Special Publication 460, Washington

[3] Goldstein, J. I, et. al., Scanning Electron Microscopy and X-ray Analysis, (2003), Kluwar/Academic Press, New York

[4] Castaing, R., Thesis, University of Paris, ONERA Publication No. 55

[5] Heinrich, K. F. J., Proc. $2^{\text {nd }}$ Nat. Conf. Electron-Probe Analysis Soc., Boston (1967), paper 2

[6] Pouchou, J. L. and Pichoir, F., Electron Probe Quantitation, eds. Heinrich, K. F. J. and Newbury, D. E., (1991), Plenum, New York

[7] Curgenven, L and Duncumb, P., Tube Investments Research Lab Report 303 (1971) 